\title{
Proximidad y afectividad \\ en las instituciones contemporáneas
}

\section{Noelia Alejandra Sierra}

Licenciada en Trabajo Social (Universidad Nacional de Buenos Aires)

Estudiante Maestría en Intervención Social

(Universidad Nacional de Buenos Aires)

Correo:noe_sierra@hotmail.com 
Resumen

En el presente artículo proponemos introducir reflexiones en torno a los desafíos que implica recomponer la institucionalidad argentina haciendo foco en el vínculo que se construye con las y los usuarios. Estas ideas generan inquietudes y obligan a analizar la intervención social en tensión entre las subjetividades actuales, la demanda de atención y los modos de acceso y efectivización de derechos. Para abordar el análisis en un primer momento desarrollaremos el concepto de institución. Luego analizaremos cierta afirmación que habla de crisis y ocaso de lo institucional para, y por último, darle lugar a dos dimensiones que pueden aportar herramientas para construir instituciones más sensibles a los cambios propios del devenir contemporáneo, nos referimos a lo que denominaremos la dimensión institucional de la afectividad y la dimensión institucional de la proximidad.

Palabras claves instituciones, subjetividad, reconocimiento, hospitalidad
Abstract

In this article we propose to introduce some reflections about the challenges involved in recomposing the Argentine institutionality, focusing on the link that is built with the users. These ideas generate concerns and force us to analyze the social intervention in tension between current subjectivities, the demand for attention and the ways to access and make those rights a reality. In order to address this analysis, we will first develop the concept of institution. Then, we will analyze a certain statement that speaks of crisis and decline of the institutional for. Finally, we want to give place to two dimensions that can provide tools to build institutions more sensitive to the changes of the contemporary world, we refer to what we call the institutional dimension of affectivity and the institutional dimension of proximity.

\section{Keywords}

institutions, subjectivity, recognition, hospitality 


\title{
Introducción
}

\author{
Por lo que a nosotros respecta "entre", era red, red de presencias. \\ El entre es un material, como la arcilla, el bosque, \\ la piedra, el hierro, los huesos o la lana \\ Fernand Deligny ${ }^{39}$
}

En la actualidad, asistimos a un proceso de reconfiguración de mandatos institucionales. Un momento de revisión de las certezas que las instituciones portaban como dadoras de sentido. Por ello consideramos necesario revisar la pregunta acerca de cómo y dónde se constituye el sujeto hoy. Y también revisar qué formatos y acciones institucionales son necesarias para las subjetividades actuales.

Dubet (2006) denomina a este proceso como el declive de las instituciones modernas. Nos referimos a la pérdida de la capacidad mediadora de las instituciones como signo de debilitamiento de su forma de trabajo sobre los otros ${ }^{40}$ y de la desarticulación de los procesos de socialización y subjetivación.

Actualmente se observa una brecha entre lo que una institución espera y lo que efectivamente se presenta en las aulas, en los pasillos, los consultorios, en las ventanillas de atención. Todo lo cierto parece desvanecerse y exige preguntarse qué hacer con estas subjetividades que ponen en tela de juicio las operaciones, visiones y procedimientos habituales para atender las problemáticas sociales actuales.

La Argentina evidenció un crecimiento económico con inclusión social en el período 2003-2015 que se tradujo en el mejoramiento de la distribución de la riqueza ${ }^{41}$. No obstante, podríamos señalar dos cuestiones en este proceso.

En primer lugar, la premisa que lúcidamente explicitan Danani, Arias, Chiara y Gluz acerca de que las reformas del periodo men-

39 En: Lo Arácnido u otros relatos. Buenos Aires: Cactus. 2015.

40 El trabajo sobre los otros refiere al conjunto de actividades profesionales que participan en la socialización de los individuos (Dubet, 2006).

41 Sugerimos consultar el siguiente artículo para profundizar y ampliar este punto de discusión: Danani, C., Arias, A., Chiara, M. y Gluz, N. (2018). Instrumentos, estrategias, apoyo y oposición en la contra-reforma de política social argentina, 2002-2015. Revista MERCOSUR de política social, 2, 132-150. Disponible en http://revista.ismercosur.org/index.php/revista/article/view/71/31 
cionado no conmovieron la estructura histórica de la protección social, pero sí desafiaron su funcionamiento. Refieren al respecto que "la estrategia de habilitar el acceso a instituciones de la seguridad social a través del pago subsidiario, trajo al campo de las políticas de protección y bienestar el desafío de la socialización” (2018 145). Las autoras señalan que, si bien las políticas del periodo mencionado pretendían recomponer la institucionalidad, en su análisis advierten debilidades, por ejemplo, la creciente elección de servicios privados (educación, salud) y por tanto el distanciamiento de "lo público". La fuga poblacional hacia prestaciones privadas genera que permanezcan en las prestaciones público estatales sólo quienes no pueden marcharse a otras instituciones corroyendo la posibilidad de concretar "una socialización compartida". Principal activo de las instituciones públicas (2018:147).

En segundo lugar, en la Argentina se avanzó en el plano normativo-legal de manera progresiva, reconociendo derechos ${ }^{42}$, pero, sin embargo, dichos progresos, no se ven cristalizados en las prácticas y en el orden de lo institucional. Solo para poner unos ejemplos podemos mencionar las disparidades en la implementación de ESI en las escuelas, o la falta de consolidación, y en algunos casos de creación, de Sistemas de Protección Integral de Niños, Niñas y Adolescentes en diferentes provincias del país.

En este sentido consideramos necesario reflexionar alrededor de la dimensión política institucional, que nos invita a considerar la tensión entre las subjetividades contemporáneas y los dispositivos existentes. Dicha tensión interpela la intervención en sus modos clásicos de atención a los problemas y amerita una re-interpretación acerca de las significaciones que adquiere lo público, en tanto lo común, o lugar donde se construye interés general.

En esta línea se presentan las siguientes preguntas: ¿estamos vivenciando el declive de un tipo particular de institucionalidad? ¿Hacia qué nuevos modelos avanzamos? ¿Qué modificaciones son urgentes

42 Por ejemplo, la Ley de Protección de Derechos de los Niños, Niñas y Adolescentes, la obligatoriedad de la escuela media, la Ley de Salud Mental, Ley de matrimonio igualitario, etc. 
para proteger a quienes más necesitan de las instituciones? ¿De qué modo una trama institucional ofrece espacios continentes donde sea posible desplegar deseo y afectividades?

Para avanzar en el análisis e intentar comprender las complejidades de la trama institucional en la actualidad, en un primer momento definiremos y desarrollaremos el concepto de institución y alguna de sus implicancias. Luego analizaremos las referencias a la crisis y ocaso de lo institucional para, por último, darle lugar a dos dimensiones que a nuestro entender aportan herramientas para construir instituciones más sensibles a los cambios propios del devenir contemporáneo: nos referimos a lo que denominaremos la dimensión institucional de la afectividad y la dimensión institucional de la proximidad.

\section{Mutaciones institucionales}

Para pensar la institución partimos de Dubet (2006). Para dicho autor la institución es definida por su capacidad de hacer advenir un orden simbólico y de formar un tipo de sujeto ligado a este. En este sentido, la Iglesia, la Escuela, la Familia o la Justicia son instituciones que inscriban un modo particular de relaciones, que establecen posiciones y funciones en la subjetividad de los individuos. Instituyen valores, símbolos, o, en otras palabras, una naturaleza social en la naturaleza "natural" de los individuos. Según esta acepción, la noción de institución no designa solamente un tipo de aparato o de organización, sino también un tipo específico de socialización y de trabajo sobre el otro. "Las instituciones por lo tanto no son, pues únicamente "hechos" y prácticas colectivas, sino también marcos cognitivos y morales dentro de los cuales se desarrollan los pensamientos individuales Douglas en Dubet, 2006 30).

Ahora bien, como mencionamos en la introducción, en los últimos años se está produciendo un proceso que ha sido llamado por diversos intelectuales como "el declive de la institución moderna" (Dubet, 2006; Martuccelli, 2006; Kessler y Merklen, 2013). 
Con el advenimiento de la modernidad tardía ${ }^{43}$, se empieza a cuestionar la idea de un sujeto representado por las instituciones en sentido clásico y sus marcos de socialización tradicional. Se evidencian transformaciones en su función de sostenimiento, sociabilización e incluso también en las respuestas a las nuevas demandas sociales.

Retomando a Dubet, el declive del programa institucional es un signo, entre otros, de la larga mutación de los relatos de la modernidad: "la cohesión social ya no se basa en la adhesión supuestamente unánime a valores comunes ni a una definición trascendente y compartida del bien: ya no hay un dios común, ni un soberano sagrado ni demasiada autoridad tradicional" (2011:85). En consecuencia, los patrones institucionales se presentan debilitados y en pleno proceso de revisión. En ese marco las y los sujetos atravesados por contextos de inestabilidad ven afectados sus modos de socialización y subjetivación, impactando a su vez en los recorridos biográficos. Señala Dubet: "en un mundo que llevaba en sí la pluralidad de valores, la promoción del espíritu crítico y el derecho de los individuos a determinarse el gusano ya estaba en el fruto (2006:421). En este sentido podemos decir que el declive de las instituciones forma parte de la modernidad en sí y no solo como una mutación o crisis del capitalismo.

La caída de las instituciones proviene de la pérdida del monopolio de los modos y los formatos de las viejas instituciones. Parecería que en épocas donde el sujeto puede elegir sin tantos cuestionamientos qué tipo de familia ser, qué religión practicar, cuántas y cuáles vacunas aplicarse, se evidencia (por parte de quienes trabajamos en ellas y quienes acuden a ellas en búsqueda de sostenes) una mutación de la "esencia" de la función social. Dubet, junto a Martuccelli, afirma que "el esquema se ha dado vuelta de la "cima" hacia "abajo" (...) Los procedimientos de ajuste de las conductas son una actividad social, y no ya un dato "natural" de la organización a través de su sistema de roles" (2000:231). Vemos entonces que los vectores que cobran

43 Resulta importante mencionar que consideramos más oportuno hablar de modernidad tardía que de posmodernidad ya que como afirma Dubet no se evidenciaría una ruptura brutal de un modelo al otro sino una suerte de prolongada implosión surgida de la modernidad en sí cuando se prosigue el proceso de racionalización, de desencanto y diversificación de la vida social y de sus representaciones (Dubet 2006:64). 
centralidad son: la experiencia social del sujeto, la reflexividad y la construcción identitaria permanente, en lugar de lo estatutario, las reglas y los roles preestablecidos.

Frente a este panorama, los individuos montan sus estrategias vitales por medio de recursos sociales muy desiguales (Martuccelli, 2007). A diferencia de lo que sostenía la sociología clásica, que proponía integrar al sujeto a las estructuras dadas a como dé lugar, hoy, en cambio, se estaría visualizando una potente exigencia a que el sujeto se construya a sí mismo, a actuar sobre la subjetividad individual con el fin de producirla como tal. Las políticas del individuo se centran hoy en la producción del sujeto individual y responsable de sí mismo (Merklen, 2013).

De esta manera se valoriza un sujeto activo, gustoso por los cambios, desentendido de las estructuras estables consideradas, en líneas generales, como procesos anquilosados, rígidos y sin brillo. Se estructuran fuertes mandatos de activación y de productividad. Un sujeto emprendedor, empresario de su propio devenir, activo de su éxito, incluso responsable de los riesgos.

En la misma línea, Bauman (2010) sostiene que el riesgo principal de la "modernidad líquida" es que cada individuo se encargue de restituir las endebles condiciones de lo social. Pero a la vez hay que tener en cuenta que a la flexibilización de las dinámicas estructurales se suma una tendencia a la desnormativización de la vida social, entendida como una demanda social de liberalización, alejándose de toda atadura institucional que pudiera constreñir al individuo, con los riesgos que eso implica en términos de una vida con otros. No obstante, la paradoja que caracteriza hoy fuertemente el fenómeno de la socialización, la única certeza que parece quedar es aquella que, limitada y modesta, señala que en el fondo de cada uno es donde hay que encontrar la verdad del propio recorrido identificatorio (Jacinto, 2009).

A nuestro criterio que el sujeto quede a merced de ser el único narrador de su existencia sin institucionalidad que acompañe es una alerta, y más si consideramos que esto podría derivar en un debilitamiento del soporte de la dimensión colectiva como acto subjetivante. 


\section{La experiencia y los agenciamientos colectivos: de la identificación de masa a la experiencia como acto subjetivante}

Frente al panorama institucional en declive, evidentemente la integración social no se está realizando exclusivamente por medio de las instituciones modernas (la familia, la escuela, la fábrica, etc.). El proceso que se está viviendo respecto a la mediación institucional ya no asegura un pasaje lineal para la socialización mediante la adscripción a normas universales.

La crisis institucional debilita considerablemente esta representación. Hoy el sujeto se presenta como "dueño de sí mismo" con el imperativo social y subjetivo de definirse según sus propios marcos de referencia.

Los individuos, siguiendo a Martuccelli, "se sienten - y soncada vez más movilizados de manera coercitiva en la sociedad y al mismo tiempo sienten que son, cada uno de ellos, irreductiblemente distintos (2017:96). Es decir, el sujeto habita una tensión entre el reconocimiento de su singularidad como única e irrepetible y al mismo tiempo se percibe indisociado de la vida social.

Martuccelli y Dubet refieren al respecto:

la integración social sin duda ha perdido su coherencia y su claridad, pero no hay seguridad que tengamos que abandonar el modelo. Dos fenómenos corroboran esta última afirmación. En primer lugar, la experiencia individual proyectada por la desinstitucionalización sigue siendo una experiencia social inscripta en relaciones sociales desiguales, son pruebas sociales. Por otro lado, los individuos tratan de inscribirse en identidades colectivas y en raíces que movilizan para construir está individualidad, de esta manera dan lugar a otros tipos de relaciones sociales" (Dubet y Martuccelli, 2000:233).

Habitar esta tensión es un desafío de nuestra época que da pie para preguntarse ¿qué formatos institucionales son necesarios para las subjetividades actuales? ¿De qué modo la individualidad es sos- 
tenida por coordenadas institucionales que la pueden alojar sin sobre-determinarla?

A esta altura podemos afirmar que los formatos institucionales clásicos tal cual se presenta - dan cuenta de un agotamiento. Las y los trabajadores institucionales con más conciencia de tal agotamiento o más negación ensayan nuevas coordenadas, revisan sus normativas a la luz de las percepciones que los sujetos tienen de éstas, experimentan nuevos encuentros con él/la otra/o. Problematizan los accesos construidos (Wagner, 2013) y los itinerarios por los que los sujetos transitan en la búsqueda del acceso a derechos. En definitiva, se busca construir nuevos puentes que permitan disminuir los malestares y desavenencias presentes en los discursos y las vivencias de unos y otros.

En este sentido, consideramos necesario prestar atención a dos dimensiones que pueden aportar herramientas para construir instituciones acordes a los modos de socialización en el declive: nos referimos a lo que denominaremos la dimensión institucional de la afectividad y la dimensión institucional de la proximidad.

\section{La dimensión institucional de la afectividad. Tensiones actuales, entre la experiencia y los agenciamientos colectivo}

En tiempos de declive de las instituciones y sus consecuencias en el lazo social, se producen relaciones paradójicas entre éstas y el sujeto, ya que parece que el sujeto no solo es hablado por las instituciones, sino que también él las hace hablar. Es construido y, a su vez, construye nueva institución. En este movimiento el valor de la experiencia vivida cobra una centralidad diferente. Dubet entiende por experiencia social "a la cristalización, más o menos estable, en los individuos y los grupos, de lógicas de acción diferentes, a veces opuestas, que los actores deben combinar y jerarquizar a fin de constituirse como sujetos" (2011:117). En este sentido consideramos que son necesarios nuevos análisis que destierren ideas en torno a conceptos unívocos, cartas echadas de antemano o casilleros estándar. Coincidimos nuevamente con el autor cuando afirma que la representación clásica de 
la sociedad debe sustituirse por otra que no destruya el lazo social entre el actor y el sistema, sino que lo multiplique (Dubet, 2011:123).

Si contemplamos, por ejemplo, el movimiento "Ni una Menos" ${ }^{44}$, la experiencia vivida relacionada con el dolor, el encuentro y las reivindicaciones posibilitó, entre otras cosas, la consolidación de un colectivo o una masa. En lo singular del padecimiento y el deseo de que otros modos vinculares sean posibles se generó en primer lugar empatía y luego sororidad. Hoy el lazo social tiene la fuerza de la fraternidad entre las y los sujetos que, siendo desconocidos los acerca la semejanza, los ubican en una común unión, y por lo tanto los iguala. En esas expresiones pareciera que el "yo" se hace "universal" en ese orden y no al revés. A su vez, en estas nuevas relaciones sociales, es desde donde se busca proyectar la identidad, se defienden posiciones sociales, se priorizan valores, se construyen otros sentidos. Podríamos pensar que la integración social pasa a ser una actividad persistente a cargo no solo de las estructuras establecidas a priori, sino también de las y los sujetos, su acción y su reflexión.

Estudiar los fenómenos colectivos desde las experiencias de los individuos estimula una forma particular de la imaginación sociológica (Mills, 1967 en Martuccelli, 2017). Implica una nueva manera de sintetizar experiencias personales, cuestiones de estructura social y movilizaciones históricas. En este sentido, incorporar en nuestro análisis y trabajo institucional la dimensión de la experiencia nos trae la necesidad de, por un lado pensar que es lo común y que es lo singular y como se imbrican socialmente, y por otro, el papel central del deseo, la imperiosa y necesaria tarea de acompañar a sujetos esperanzadamente deseosos (Ulloa, 1995) y que las tramas institucionales habiliten afectos y aspiraciones donde las y los sujetos puedan percibirse cuidados en el despliegue de su potencia. Hablamos de escuelas, centros juveniles, espacios artísticos, instituciones de culto, que dejen

$44 \mathrm{Ni}$ una menos es una consigna que dio nombre a un movimiento feminista surgido en Argentina, el 3 de junio de 2015. Es un colectivo de protesta que se opone a la violencia contra la mujer y su consecuencia más grave y visible. Innumerables mujeres de todo el país se organizan y salen a la calle a gritar "Ni Una Menos". 
una huella subjetiva ${ }^{45}$ significativa en quienes las habitan, que en algunos casos pudieron, de algún modo, dar lugar a sus necesidades y a la vez modificar las estructuras para garantizar derechos.

Las experiencias institucionales que dan lugar a la dimensión de la afectividad pueden resultar incómodas, ya que invitan a pensar de otro modo en pos de alojar a un otro que siempre es radicalmente otro/a. La experiencia vivida tiene en la actualidad la fuerza de la heterogeneidad que se pregunta qué es lo que fraterna qué hay de común. En la experiencia vivida se proyecta lo intransferible del deseo, los nuevos sentidos que buscan encontrar formatos políticosinstitucionales que la representen sin sobredeterminarla. Así lo explicita Martuccelli cuando analiza la nueva dinámica social de la modernidad: "la vida individual es siempre en común; la irreductible singularidad existencial de cada cual solo es posible à partir del común del cual participa” (2017:101).

La experiencia se sustenta fundamentalmente en el relato de lo acontecido. Un relato que expone, denuncia, informa, confraterniza, busca empatizar o provocar, polemizar o comulgar. Este relato puede encontrar escucha en una asamblea o corte de calle, en un aula, en una iglesia o puede ser publicable en una red social, también en la musicalidad de un reggaeton, en la pluma de una poesía o de un graffitti que gana la calle... pareciera que el relato de la experiencia vivida contemporánea se presenta huérfano de anclajes y representantes institucionales clásicos, pero con la potencia de lo nuevo que irrumpe y requiere de otros modos de escuchar, observar e interpretar.

Ahora bien, en este plano del análisis nos interesa interrogar ¿qué de la experiencia puede ser institucionalizado?, ¿Con qué sentidos? ¿Cuándo y de qué modo habría que hacerlo? En definitiva, ¿qué es institucionalizar la experiencia? No se encuentran certezas acerca de este punto, sí nos atrevemos a decir que en la irrupción de la experiencia social hay nuevos anclajes que pensar y que resulta imperativo

45 Para una mayor profundización sobre el concepto de huellas institucionales en jóvenes estudiantes del nivel secundario recomendamos consultar el artículo recientemente publicado de Napoli y Richter (2019). 
en estos tiempos la (de) construcción de dispositivos institucionales que reconozcan estas nuevas expresiones subjetivas sin necesidad de renunciar a intervenir sobre ellas (Cantarelli, 2014).

En tiempos de caída de representaciones institucionales totalizantes con las consecuencias que ello trae en la socialización y el relato de las experiencias vividas, consideramos importante repensar la política de las instituciones en torno a la dimensión afectiva. Nos referimos a imaginar de manera urgente, nuevas conformaciones institucionales que puedan socializar y subjetivar dándole lugar a la trama de deseos, afectividades, gestualidades y palabras para que las y los sujetos encuentren en las ellas (o continúen encontrando, como en algunos casos) un lugar donde percibir protección, igualdad, y la posibilidad de lazo con otros.

\section{La dimensión institucional de la proximidad. El reconocimiento y la hospitalidad como acto subjetivante}

En la misma línea del desarrollo del punto anterior, otro aspecto a tener en cuenta a la hora de reflexionar sobre la institucionalidad en la vida contemporánea, es lo que llamaremos la dimensión institucional de la proximidad.

Una institución próxima es una institución empática y presente. Nos referimos a una presencia, como indica Kantor, no como un mero estar ahí, sino teniendo en cuenta la forma en que se despliega y los significados que adquiere (2008 71). La institución como una instancia próxima que habilite espacios y momentos de pertenencia, hospitalidad y reconocimiento.

Derrida (2000) propone volver a la pregunta acerca del otro, en su libro "La hospitalidad", e insiste acerca de cómo alojamos al otro, al diferente, al extranjero, al extraño. Para el filósofo la hospitalidad pura consiste en "acoger al arribante antes de ponerle condiciones, antes de saber y de decirle o preguntarle lo que sea, ya sea su nombre o ya sean unos <papeles> de identidad"; implica además que nos dirijamos a él, es decir que lo nombremos y reconozcamos su nombre propio. Para dicho autor la hospitalidad consiste en hacer todo lo po- 
sible para dirigirse al otro, evitando que las preguntas se conviertan en una condición, una inquisición policial, un fichaje o un simple control de fronteras. Otorgar un lugar al otro, ser hospitalarios implica no solo agrandar el hogar sino refundar muchos de los sentidos desde los cuales ese hogar se construyó. Implica un encuentro dialógico que amalgame teorías, ideologías, discursos, prácticas, certezas institucionales y contingencias apócales.

Es importante en este punto la pregunta sobre cómo alojamos al otro, qué modos adquieren nuestras instituciones para que las y los sujetos encuentren en ellas algo de sosiego, un lugar propio donde anclar el deseo, un momento para ser y estar. Por ello, resulta bien interesante la distinción que sostiene Derrida (1997) retomada por Rouam (2017) en relación al posicionamiento que se asume frente al otro extraño y ajeno. Nos referimos a la diferencia entre tolerancia y hospitalidad.

La tolerancia proviene del latín "soportar", aparece del lado de la tradición cristiana. Postula una lógica donde, aunque el otro sea diferente a mí, debo "tolerarlo" ya que el otro es un prójimo. Al volverlo un prójimo lo vuelvo próximo, alguien de los propios, así es que el otro termina de perder su otredad (Rouan, 2017). Señala Derrida: "si yo creo ser hospitalario porque soy tolerante, es que deseo limitar mi acogida, mantener el poder y controlar los límites de mi casa, de mi soberanía, de mi lengua, de mi cultura, de mi religión (2000:3). En cambio, la idea de hospitalidad implica un recibimiento al extranjero, pero no condicionado. El filósofo argumenta:

(...) parece dictar que la hospitalidad absoluta rompe con la ley de la hospitalidad como derecho o deber, con el pacto de la hospitalidad. Para decirlo en otros términos, la hospitalidad absoluta exige que yo abra mi casa y que dé no solo al extranjero sino al otro absoluto, desconocido, anónimo, y que le dé lugar, lo deje venir, lo deje llegar y tener lugar en el lugar que le ofrezco, sin pedirle ni reciprocidad ni siquiera su nombre (Derrida, 1997:31). 
De modo que la alarma pareciera ser que la proximidad institucional desde el discurso de la tolerancia no anule al otro/a sino que, contrariamente, potencie el devenir singular de las y los sujetos que acuden a las instituciones en busca de soportes seguros y confiados desde donde proyectarse.

Ensayar la hospitalidad institucional es preguntarnos en un primer momento, qué lugar damos al extranjero. Esta pregunta permitirá revisar las particularidades que adquieren nuestros procedimientos habituales para sostener intervenciones alojantes. Es la posibilidad para que quien oficie de huésped haga preguntas, habilite interrogantes y que en las respuestas (siempre provisorias) se encuentren otros modos de reconocimiento.

Proyectar la hospitalidad en las instituciones tiene que ver con revisar el vínculo que tenemos con el sujeto contemporáneo y pensar si será posible la organización de un principio de proximidad donde estar junto al otro/a conmueva algo de mi instituido y abra a la posibilidad de un nuevo instituyente. La construcción de un proceso vincular "entre" los usuarios y las y los agentes institucionales en la actualidad es una tarea artesanal, es decir sin moldes ni recetas a priori, donde la humanización de la intervención a raíz del conocimiento mutuo y la práctica del reconocimiento (Honneth, 2000 en Dubet, 2006) debería ser nuestro faro conceptual, empírico y político si se desea que las instituciones protejan a las y los sujetos:

El reconocimiento no es una norma de justicia objetiva, universal y mensurable. Deriva de una esfera de justicia subjetiva que varía de individuo en individuo y conforme a quien lo distribuya, pero es una norma más de exigente por cuanto tiene como función reducir las tensiones entre la igualdad fundamental de todos los individuos y sus desigualdades de desempeño y situación. El sujeto desea que lo reconozcan (Dubet, 2006:95).

En conclusión, en la medida que las instituciones intermedien y ensayen acciones desde la hospitalidad, la proximidad y el valor del reconocimiento en tanto actos subjetivante pueden ser dimensiones para nuevas y pujantes experiencias habilitantes de derechos. 


\section{Notas finales}

Asistimos a un proceso de desapego institucional en el cual repensar los modos que tenemos en la experiencia con y sobre los otros es de una importancia enorme. Precisamente, si tal como afirma Dubet lo institucional está en tensión, habitar y reflexionar en esta tensión nos permitirá encontrar nuevos modos de ser institución.

En la construcción de vínculos próximos, fraternos y de amparo se alza la posibilidad de una intervención que reconozca y habilite derechos. La posibilidad que tenemos las y los contemporáneos de avanzar hacia modelos institucionales más sensibles dependerá de cuánta lectura de contexto tendremos poniendo en valor dimensiones como el reconocimiento de la diversidad social y de las sexualidades, el lugar para las afectividades en los encuentros, la potencia de la experiencia en vínculo con las expectativas colectivas, el enaltecimiento de la alteridad y los criterios de igualación frente a la persistente desigualdad. Aspectos todos, que permitirían encontrar lugares institucionales amorosos para el despliegue de la vida deseada.

\section{Referencias bibliográficas}

Abad, Sebastián y Cantarelli, Mariana. Habitar el Estado. Pensamiento estatal en tiempos a-estatales. Buenos Aires: Hydra, 2010.

Bauman, Zigmund (2010). Mundo consumo. Buenos Aires. Paidós.

Danani, Claudia, Arias, Ana, Chiara, M. y Gluz, N. (2018). Instrumentos, estrategias, apoyo y oposición en la contra- reforma de política social argentina, 2002-2015. Revista MERCOSUR de política social, 2, (pp. 132-150). Doi: 10.289197 (ism.2018-v2-132). Disponible en: http://revista.ismercosur.org/index.php/revista/article/view/71/31

Derrida Jacques (1997b). "El principio de la hospitalidad”. Entrevista realizada por Dominique Dombres, Le Monde, 2 de diciembre de 1997. Disponible en https:// redaprenderycambiar.com.ar/derrida/textos/hospitalidad.htm

Derrida, Jacques; Dufourmantelle, A., \& Segoviano, M. (2008). La hospitalidad. Buenos Aires, Argentina, Ediciones de la Flor.

Di Leo, Pablo y Camarotti, Ana Clara (2013). Quiero escribir mi historia. Vidas de Jóvenes en Barrios Populares. Buenos Aires, Biblos. 
Proximidad y afectividad en las instituciones contemporánea Noelia Alejandra Sierra

Dipaola, Esteban (2013). Hospitalidad y Reconocimiento: Reflexiones sobre el otro y sobre la igualdad en sociedades de consumo. Disponible en: http://diferencias.com.ar/ congreso/ICLTS2015/ponencias/Mesa\%2038/ICLTS2015_Mesa38_Dipaola.pdf

Di Napoli Pablo y Ritchter Nicolás (2019). Huellas instituciones en la subjetividad de estudiantes de educación secundaria. En: Arias, Ana y Di Leo, Pablo (Directores), "Jóvenes e instituciones. El derecho a ser en barrios populares”. Bs. As., Editorial Espacio.

Dubet, Francois (2006). El declive de la institución. Profesiones, sujetos e individuos en la modernidad. España, Gedisa.

Dubet, Francois (2011). La Experiencia sociológica. Barcelona, España, Gedisa.

Dubet, Francois y Martuccelli, Danilo (2000). ¿En qué sociedad vivimos? Buenos Aires, Argentina, Losada.

Dusell, Enrique (2012). Para una política de la liberación. Buenos Aires, Editorial Las cuarenta/Gorla.

Jacinto, Claudia y Milleanear, Verónica (2009). "Enfoque de Programas para la inclusión laboral de los jóvenes pobres: Lo institucional como soporte subjetivo”. En: Ultima Década, N³0, CIDPA VALPARAÍSO, JULIO 2009, (pp. 67-92).

Kantor, Débora (2008). Variaciones para educar adolescentes y jóvenes. 1a ed. Buenos Aires, Del Estante Editorial.

Kessler, Gabriel (2014). Controversias sobre la desigualdad. Argentina, 2003-2013. Buenos Aires, Fondo de Cultura Económica, (pp. 13 a 57).

Kessler, Gabriel (2011) Exclusión social y desigualdad ¿Nociones útiles para pensar la estructura social argentina? En: Laboratorio/on line, Revista de estudios sobre cambio social, año XII, número 24, Verano 2011. Buenos Aires, Instituto G. Germani, Facultad de Ciencias Sociales, Universidad de Buenos Aires.

Martuccelli, Danilo (2017). La nueva dinámica de la condición social moderna. En: Revista de Sociología 32(1), (pp. 89-105). DOI: 10.5354/0719-529x.2017.478874.

Rouan, Malena. La ética de la Hospitalidad. En: Revista Clepios. Disponible en: http:// clepios.com.ar/74/trabajos/la-etica-de-la-hospitalidad/

Ulloa, Fernando (1995). Novela clínica psicoanalítica. Paidos. Buenos Aires.

Wagner, Alejandra (2013). Volviendo sobre el concepto de accesibilidad en diálogo con el territorio. Apuntes de experiencias extensionistas, en clave de investigación. En: Testa, Ma. Cecilia (Org). Trabajo Social y territorio. Reflexiones sobre lo público y las instituciones. Buenos Aires, Editorial Espacio.

Recepción: 20/03/2020

Aceptación: 10/08/2020 